\title{
A Influência da Bichectomia na Agradabilidade Facial
}

\author{
The Influence of Bichectomy on Facial Pleasantness
}

\author{
Lucia Gloria Diana Aguilar Pizzurno; ; Ana Claudia de Castro Ferreira Contia; Marcio Rodrigues de Almeida ${ }^{\text {aa }}$ Paula \\ Oltramaria; Thais Maria Fernandes Polettia; Renata Rodrigues de Almeida-Pedrin*a
}

aUnopar, Programa de Pós-Graduação Stricto Sensu em Odontologia. PR, Brasil.

*E-mail: renatinha@uol.com.br

\begin{abstract}
Resumo
A beleza pode ser definida como uma combinação de qualidades que dão prazer aos sentidos ou a mente. Cirurgiões plásticos acreditam que o volume da bochecha é um importante determinante da beleza facial e aparência jovem. Na Ortodontia, não é suficiente apenas perceber o que interfere no sorriso, é necessário diagnosticar o que se encontra fora da normalidade, para que se possa estabelecer um plano de tratamento. Assim como nos problemas funcionais seguimos condutas que nos levam ao diagnóstico das anomalias, os problemas estéticos também necessitam de parâmetros para que encontremos os defeitos. Nesse âmbito, as investigações científicas sobre os critérios utilizados pelo ortodontista e pacientes para definir um sorriso ou uma face como esteticamente agradável poderiam edificar uma sólida escada para o entendimento das diferenças entre a percepção do profissional e a do paciente. Durante a execução do tratamento, a consulta de pesquisas cientificas que norteiem a situação favorece seu decurso. Nortear a identificação e manejo no diagnóstico e indicação adequada da bichectomia foi o objetivo desta revisão de literatura realizada nas bases de dados científicas National Library of Medicine, Washington, DC (MEDLINE: Pubmed) e Scientific Electronic Library Online (SciELO). Foram recuperados 1710 artigos científicos, que após seleção resultaram em 48 artigos relacionados à descrição e manejo no diagnóstico e indicação adequada da Bichectomia, escolhidos para elaboração desta revisão de literatura. A existência de materiais de fácil consulta com embasamento científico adequado para orientação aos pacientes na tomada de decisões enquanto a Bichectomia favorece manejo do tratamento.
\end{abstract}

Palavras-chave: Bichectomia. Gordura de Bichat. Estética. Diagnostico. Ortodontia.

\begin{abstract}
Beauty can be defined as a combination of qualities that give pleasure to the senses or the mind. Plastic surgeons believe that cheek volume is an important determinant of facial beauty and youthful appearance. In orthodontics, it is not enough to just understand what interferes with the smile, it is necessary to diagnose what is out of the ordinary, so that a treatment plan can be established. Just as in functional problems we follow conducts that lead us to the diagnosis of anomalies, aesthetic problems also need parameters in order to find the defects. In this context, scientific investigations on the criteria used by orthodontists and patients to define a smile or face as aesthetically pleasing could build a solid ladder for understanding the differences between the professional's and the patient's perception. During the execution of the treatment, the consultation of scientific research that guides the situation favors its course. To guide the identification and management in the diagnosis and adequate indication of bichectomy was the objective of this literature review carried out in the scientific databases National Library of Medicine, Washington, DC (MEDLINE: Pubmed) and Scientific Electronic Library Online (SciELO). 1710 scientific articles were retrieved, which after selection resulted in 48 articles related to the description and management in the diagnosis and adequate indication of Bichectomy, chosen for the elaboration of this literature review. The existence of easy-to-consult materials with an adequate scientific basis to guide patients in making decisions while Bichectomy favors treatment management.
\end{abstract}

Keywords: Bichectomy. Buccal Fat Pad. Aesthetics. Diagnostics. Orthodontics.

\section{Introdução}

Há poucas visões mais agradáveis do que um rosto bonito. Faces atraentes ativam centros de recompensa no cérebro, e eles provocam atribuições de personalidade positiva. O estereótipo: "O que é bonito é bom" em uma variedade de configurações não é surpreendente, portanto, filósofos, cientistas, e pessoas comuns têm buscado incessantemente descobrir o que torna um rosto atraente e harmonioso (RHODES, 2006). As percepções de beleza facial são multifatoriais incluindo características genéticas, ambientais e culturais distintas (NAINI, 2006).

A beleza pode ser definida como uma combinação de qualidades que dão prazer aos sentidos ou a mente. A estética é o estudo da beleza e, em menor escala, o seu oposto, o feio (NAINI, 2006). Os dentistas geralmente reconhecem que a necessidade de tratamento das más oclusões pode se basear em outros fatores que não incluem o comprometimento da função fisiológica ou o potencial de perda da função. A motivação para buscar tratamento ortodôntico parece estar fortemente relacionada à percepção dos indivíduos sobre até que ponto sua aparência dentofacial se desvia das normas socioculturais (TEDESCO, 1983).

Uma face jovem e atraente é caracterizada por uma bochecha bem contornada e anteriormente projetada, com o envelhecimento, essa área perde gradualmente o volume, principalmente devido a redução do coxin gorduroso e, 
em menor grau, pela reabsorção óssea moderada (BRAZ; SAKUMA, 2012). Cirurgiões plásticos acreditam que o volume da bochecha é um importante determinante da beleza facial e aparência jovem (BERGMAN, 1999).

A análise morfológica da face é o principal recurso diagnóstico para determinação do padrão facial que, por sua vez, remete a protocolos de tratamento e prognósticos específicos em diferentes faixas etárias. Todo ortodontista visa proporcionar aos pacientes tratamentos não só com resultados satisfatórios quanto a oclusão e função, mas também melhorar a estética facial, através de um correto diagnóstico, considerando a morfologia facial (REIS et al., 2011).

$\mathrm{Na}$ Ortodontia, não é suficiente apenas perceber o que interfere no sorriso, é necessário diagnosticar o que se encontra fora da normalidade, para que se possa estabelecer um plano de tratamento. Assim como nos problemas funcionais seguimos condutas que nos levam ao diagnóstico das anomalias, os problemas estéticos também necessitam de parâmetros para que encontremos os defeitos. Se pensarmos em estética relacionada à face, através de pesquisas, podemos encontrar os pontos de congruência e diferenças entre o paciente e o profissional e, com isso, desenvolver um guia para usar como parâmetro no momento do diagnóstico (NORMANDO, 2012).

A valorização e busca pela estética se mostram como forte tendência na sociedade atual. No campo de atuação do cirurgião-dentista, o referencial estético, tradicionalmente, é o sorriso. Contudo, a avaliação do padrão facial, linhas de expressão, contornos faciais e possíveis melhorias tem se tornado uma prática rotineira entre os dentistas. Na construção da imagem pessoal, há muitos aspectos a serem levados em consideração, no entanto não devemos esquecer a importância ou o papel principal que o terço médio da face representa devido à sua localização (STEVÃO, 2015).

A busca pelo tratamento ortodôntico ao longo dos anos acrescenta pontos a favor da estética como principal motivo, nesse sentido, cabe ao profissional conhecer o que considera o paciente um rosto agradável. Essa situação revela que, ao planejar um tratamento, ele deve ser apoiado por uma extensa análise, não apenas dos problemas cefalométricos e funcionais, mas também dos requisitos estéticos do paciente (MOROSINI et al., 2012).

Esta revisão é uma forma de se ampliar os conhecimentos a respeito dos princípios estéticos, incluindo a bichectomia, aplicados a face, fundamentais na avaliação de sua morfologia, planejamento e tratamento ortodôntico.

\section{Desenvolvimento}

\subsection{Metodologia}

Uma revisão narrativa foi realizada nas bases de dados científicas National Library of Medicine, Washington, DC (MEDLINE: Pubmed) e Scientific Electronic Library Online (SciELO), Lilacs e Capes contemplando todos os tipos de artigos científicos. A filtragem apresentou como critérios: disponibilidade de texto integral, estudos realizados em humanos e publicados nos idiomas Inglês, Espanhol ou Português (a busca foi no mês março de 2020). Ao finalizar as pesquisas em cada base, as referências duplicadas foram excluídas. Os termos utilizados nas buscas foram "bichectomia", "gordura de Bichat", "estética facial", "ortodontia”, “diagnostico". A partir da leitura dos títulos 1710 artigos, a filtragem inicial dos estudos foi realizada, excluindo-se os artigos que não atendiam a descrição e que não forem relevantes e pertinentes na matéria enquanto a conceito, agradabilidade facial, diagnóstico e tratamento de Bichectomia, obtendo-se um total de 48 artigos que foram selecionados para a compilação de dados necessários para a investigação bibliográfica.

\subsection{Revisão de literatura}

O conceito de estética facial é objeto de curiosidade desde os tempos antigos, atualmente continua a se expandir e se transformar independentemente da diversidade étnica, com choque em todos os estratos sociais (CERRATI; THOMAS, 2017).

Definir a beleza facial através de fórmulas numéricas é um método incompleto de entender o leque de possibilidades na escala de agradabilidade facial. Ou seja, podemos encontrar rostos e dentes perfeitos sem necessariamente ser harmoniosos para os outros. Olhando para as faces objetivamente, observase uma infinita variação racial, familiar ou individual e oclusões dentárias normais são encontradas numa ampla variedade de faces. Quando se estuda a face, nós vemos o que vemos e não aquilo que fomos levados a acreditar que deveríamos ver. A multiplicidade de fatores envolvidos na estética facial certamente depõe contra qualquer padronização, seja ela geométrica ou de outra forma (WILSON, 1957).

Até a segunda metade do século XVIII, o termo "estética" não possuía o mesmo significado que se reconhece atualmente para designar 'a filosofia do belo', com investigação teórica. Porém o fato existia antes do nome, pois a reflexão sobre a beleza e as belas artes começaram entre os pensadores helênicos e à época de Sócrates, ou até mesmo com os filósofos anteriores. Não há nenhuma definição de beleza que tenha aceitação universal. Entre os antigos, a teoria fundamental do belo baseava-se nas noções de ritmo, simetria e harmonia das partes ou, em outras palavras, com a fórmula geral da unidade na variedade. Entre os modernos, encontrase mais intensamente a ideia de significação, expressividade, subjetividade de tudo o que há na vida, aquilo que se expressa com característica individual para a percepção dos sentidos ou da imaginação (CADENA; GUERRA, 2006).

A estética é o estudo racional da beleza, da possibilidade de sua definição, à diversidade de emoções e sentimentos que ela suscita no homem. Essa definição, aparentemente simples, do significado da palavra estética, que pode ser encontrada em vários dicionários, esconde um sentido extremamente 
amplo. O estudo e a busca de seu significado real no mundo ocidental remontam aos filósofos gregos como Platão, Aristóteles e Plotino. A complexidade do termo estética é o resultado de várias teorias filosóficas que buscaram uma explicação consistente para seu significado. A palavra estética veio para designar o campo geral da estética, que incluía todas as categorias pelas quais os artistas e pensadores haviam demonstrado interesse, como trágico, sublime, gracioso, risível, humorístico etc.; poupando a palavra beleza para esse tipo especial, caracterizado pela harmonia, pelo senso de medida, pela fruição calma e serena. A estética é, então, esse tipo de reformulação de toda a filosofia em relação à beleza. E, considerando a complexidade do campo estético, surge o dilema criado pela tentação irracional: a beleza está nos olhos de quem vê ou é intrínseca ao objeto? Embora essa questão não possa ser resolvida de maneira fácil, pois trata-se de uma abordagem filosófica que cada indivíduo interpreta à sua maneira, ela pode e deve ser auxiliada em sua concepção, especialmente para ortodontistas que trabalham com estética dentofacial (CAMARA, 2012).

A imagem corporal parece influenciar o desenvolvimento de uma pessoa. Mueser et al. (1984) afirmaram que a face é um preditor ligeiramente mais importante de atratividade geral do que o corpo. Além disso, foi relatado que os olhos, a região oral e a face contribui significativamente para a atratividade facial geral. No entanto, foi sugerido que o resto do rosto parece ser uma sugestão mais influente que a estética dental na formação de impressões (TATARUNAITE et al., 2005).

A face é especialmente importante, porque tem muitos elementos morfológicos, é o principal canal de comunicação interpessoal e, na maioria das culturas, é claramente visível (KOŚCIŃSKI, 2007).

Uma das estruturas importante, o corpo adiposo da bochecha, para a agradabilidade é atratividade facial foi descrita em 1802 pelo francês Marie François Xavier Bichat, que após dissecar cerca de 600 cadáveres a descreveu como uma massa de gordura circunscrita localizada no terço médio da face, nomeando-a de bola gordurosa de Bichat, sem relevância funcional adjacente as bochechas (LOUKAS et al., 2006).

Ao descrever o espaço mastigatório como um compartimento facial que abriga os músculos temporal, masséter, pterigoideo lateral e medial, a articulação temporomandibular, o ramo da mandíbula e estruturas neurovasculares, Gaughran (1957), também incluiu o corpo adiposo da bochecha como componente desse espaço, relatando ser uma estrutura composta por um corpo principal, de onde partem quatro extensões: bucal, pterigopalatina, temporal e pterigoide.

Circunscrita no espaço de mastigação, a bola gordurosa de Bichat, se relaciona com o duto parótido e o ramo bucal do nervo facial. Ela pesa em média 3,9 g e apresenta $6 \mathrm{~mm}$ de espessura na maioria dos casos. Costuma existir uma diferença de peso de 0,51 g em média entre os lados direito e esquerdo
(CONTI, 2018; AHARI et al., 2016; FARIA et al., 2018).

Tideman, Bosanquet, Scott (1986), relataram que a extensão bucal (figura 1) da "almofada adiposa" (corpo adiposo da bochecha) repousa sobre a fáscia bucofaríngea, revestindo a superfície externa do músculo bucinador. $\mathrm{O}$ corpo principal encontra-se em profundidade ao longo de toda parte posterior da maxila e das fibras posteriores do bucinador. Segundo os autores, sua maior extensão, a "temporal", passa superiormente sob o arco zigomático para o plano temporal e se divide em duas porções: superficial e profunda. A porção superficial desloca-se entre a fáscia temporal e a superfície do músculo temporal; a profunda é estreita e transpassa entre as fibras do músculo temporal para fixar-se na asa maior do osso esfenoide, na fossa infratemporal. Assim, a extensão temporal profunda é o único processo do corpo adiposo que não pode ser facilmente separado dos tecidos adjacentes.

Figura 1 - Extensão bucal do corpo adiposo da bochecha

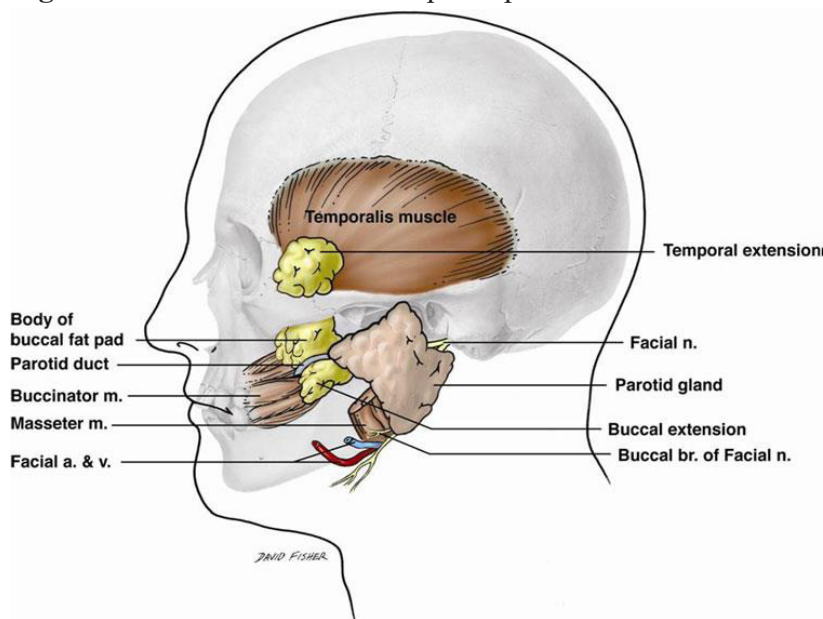

Fonte: Yousuf et al. (2010)

Ao analisarem 12 cadáveres frescos, Stuzin et al. (1990), concluíram que cerca de $30 \%$ a $40 \%$ do peso total do coxim gorduroso de Bichat é proporcionado pela porção bucal, sendo a mais larga e responsável de conferir o volume das bochechas. Sendo mais desenvolvida em lactentes, conferindo assim um contorno arredondado das bochechas (Figura 2).

Figura 2 - Porção bucal e relações com estruturas

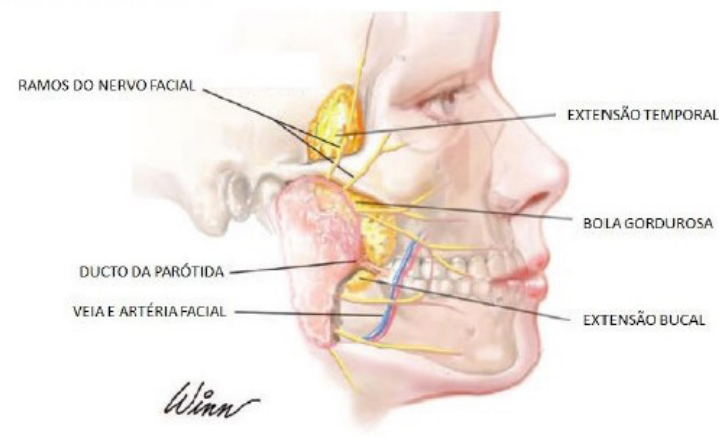

Fonte: Cohen et al. (2017).

A bichectomia consiste num procedimento cirúrgico seguro que pode ser realizado em ambiente de consultório 
e/ou ambulatório (STEVÃO, 2015; SILVA; SILVA FILHO, 2017). A exérese da estrutura anatômica requer um retalho rápido, simples e de fácil execução, que pode ser realizado com o paciente sob anestesia local e não deixa cicatrizes visíveis (DEAN et al., 2001).

A lipoplastia facial, também conhecida como bichectomia, corresponde a uma cirurgia estético funcional da face, também considerada como plástica facial, que visa reduzir o tamanho das bochechas por meio da remoção da bola gordurosa de Bichat. A cirurgia é considerada um procedimento relativamente simples, que pode ser realizado sob anestesia local. Os resultados deste procedimento vão desde o aspecto mais afinado da face, que proporciona uma bochecha menos projetada anteriormente, com acentuação da região malar e ressalte dos ângulos da região de ramo da mandíbula (AHARI, 2016).

Por meio de tomografia computadorizada e ressonância magnética Loukas et al. (2006), analisaram 80 cadáveres humanos, e constataram que o volume médio do corpo adiposo da bochecha de homens e mulheres foram, respectivamente, de $10,2 \mathrm{ml}$ e $8,9 \mathrm{ml}$, enquanto a sua espessura média foi de $6 \mathrm{~mm}$ com um peso médio de $9,7 \mathrm{~g}$. Foram examinados 80 cadáveres humanos adultos (160 hemifaces), sendo 35 mulheres e 45 homens com faixa etária 55-86 anos e idade média de 59 anos. Divididos em três grupos de acordo com sua idade: Grupo A, $<60(\mathrm{n}=38)$; Grupo B, 60-70 $(\mathrm{n}=32)$; Grupo $\mathrm{C},>70(\mathrm{n}=$ 10). A divisão dos cadáveres em três grupos foi baseada na estreita distribuição etária intragrupo e não devido a outros critérios. Havendo nitidamente um decréscimo do volume do corpo adiposo da bochecha com o avanço da idade e teve maior redução nos pacientes do sexo feminino.

A excisão cirúrgica da bola gordurosa de Bichat é chamada de bichectomia e, permite ao paciente traços mais finos e definidos conferindo uma aparência mais jovial. Além deste benefício de grande importância na área da cirurgia estética facial, a remoção pode ser justificada por causa funcional como consequência de mordedura e trauma na mucosa jugal (MATARASSO, 1991).

Um estudo recente analisou, prospectivamente, 16 pacientes submetidos ao procedimento, por meio de questionário, exame clínico e análise de fotografias. Revelou bons resultados quanto à satisfação dos pacientes, sendo que pacientes do sexo feminino demonstraram satisfação com o resultado estético, enquanto pacientes do sexo masculino notaram maior ganho funcional. Concluiu-se que a bichectomia é um procedimento seguro que permite ao paciente melhoras estéticas no contorno facial (SILVA et al., 2019).

\subsection{Discussão}

Anatomicamente, o contorno da face inferior é composto por quatro elementos: bola gordurosa de Bichat, músculo masseter, osso mandibular e a gordura subcutânea. Assim, a bola gordurosa de Bichat tem um papel importante na estética facial. Se a extensão bucal for excessiva, os pacientes podem se queixar de rosto arredondado, bochechas excessivas ou "rostos de bebês". Portanto, a remoção da bola gordurosa de Bichat, "Lipectomia bucal parcial" é apresentada como uma técnica para esculpir os ângulos faciais e melhorar a estética. O procedimento está relacionado ao conceito de "triângulo invertido" da juventude" que pode aumentar a beleza. Este conceito é definido por uma aparência facial angular resultante de uma face mais magra e com região malar alta, conferindo uma proeminência ou luminescência na região malar, além de estar intimamente relacionado com o espaço mastigatório (THOMAS; D'SILVA; BOROLE, 2012; NICOLICH; MONTENEGRO, 1997; BERNARDINO JUNIOR et al., 2008; STEVÃO, 2015; MATARASSO, 2006).

O perfil da face média é determinado pelo esqueleto facial, pela gordura de Bichat e pelo musculo masseter. Uma face com bochecha volumosa pode ser decorrente de uma hipertrofia da gordura de Bichat que pode ser devido a fibrose da região que não é produto da idade, ou a uma hipertrofia do musculo masseter, ou uma contribuição de ambos (TARALLO et al., 2018).

A técnica da bichectomia vai além, apresentando propósito funcional principalmente na redução de traumatismos crônicos mastigatórios nas mucosas jugais, decorrentes de volumes avantajados dessas estruturas anatômicas, deletérios aos tecidos bucais, passíveis de indução de lesões patológicas diversas, inclusive neoplásicas. Assim, este procedimento pode ser categorizado como estético funcional proporcionando benefícios na mastigação e não somente na estética da face (SILVA et al., 2019).

O procedimento pode ser indicado para casos com faces arredondadas ou com presença de bola de Bichat pseudoherniada (TAPIA; RUIZ-DE-ERENCHUN; RENGIFO, 2006; KRUPP, 1986; MATARASSO, 2006). Quando a pseudo-hernia é diagnosticada, o paciente mostra um pequeno contorno arredondado irregular na bochecha devido ao enfraquecimento da fáscia da bola de Bichat (MATARASSO, 2003). Pacientes com rostos arredondados mostram bochecha/ face média com plenitude apesar de ter o Índice de massa corporal adequada (MATARASSO, 1991). Nos dois casos, o objetivo do procedimento é reduzir a plenitude de face média, destacar a proeminência zigomática e mandibular, e remover qualquer assimetria dos tecidos moles (MATARASSO, 1991; ENGDAHL et al., 2012). Uma contraindicação absoluta encontrada na literatura, é em pacientes com atrofia hemifacial progressiva. Conhecida como uma doença degenerativa rara, caracteriza-se por uma atrofia facial unilateral progressivamente lenta que envolve o tecido subcutâneo, cartilagem, tecido adiposo e estruturas ósseas subjacentes (EL-KEHDY; ABBAS; RUBEIZ, 2012), onde a atrofia da bola de Bichat é um componente bem conhecido (DUBIN et al., 1989).

Embora muitos autores como, Racz, Maros, SeresSturm (1989) e Tostevin, Ellis (1995), tenham sugerido que a mastigação e a sucção sejam as principais funções da 
almofada de gordura bucal, Zhang et al., (2002) apontaram outras funções a mesma. Como os lóbulos da almofada de gordura bucal ocupam vários espaços profundos da face, a contração muscular e o movimento dos aparelhos mastigatórios e miméticos forçam os lobos da almofada de gordura bucal a funcionarem como almofadas deslizantes. Dessa forma, a almofada de gordura também serve como uma almofada contra lesões causadas por contração muscular ou trauma externo que pode ferir feixes neurovasculares faciais.

Em relação à estética, a plenitude do lobo anterior da almofada de gordura bucal, conforme destacado por Yousif et al. (1994), podem causar aprofundamento da prega nasolabial e relaxamento dos músculos miméticos, ambos associados ao envelhecimento. Além disso, a plenitude bucal sempre foi explicada pela protrusão anteroinferior da almofada de gordura bucal (LI; XU; ZHONG, 1993; MATARASSO, 1991). Entretanto, outras causas dessa plenitude incluem relaxamento dos músculos miméticos, desenvolvimento ligamentar deficiente e ruptura da cápsula da almofada de gordura bucal (ZHANG et al., 2002). Além disso, esses fatores aumentam a chance de prolapso da gordura de Bichat na cavidade oral.

Para alcançar a tão desejada beleza dos padrões de agradabilidade e atratividade facial julgados subjetivamente por cada profissional, muitos pacientes recorrem à bichectomia. Os estudos de Silva et al. (2019) e Tarallo et al. (2018) reportaram os resultados e o grau de satisfação dos pacientes com o procedimento realizado. Relataram ainda que para alcançar o efeito estético desejado, o profissional deve recorrer ao estudo minucioso, não apenas com uma avaliação clínica mas também, cercar-se de recursos auxiliares de diagnostico como imagens por ressonância magnética.

Outro questionamento envolvendo a bichectomia é em relação aos padrões faciais. Será que os resultados do procedimento são percebidos diferentemente no braquifacial, mesofacial e dolicofacial? A literatura menciona que as indicações habituais para a bichectomia são os rostos arredondados (THOMAS; D'SILVA; BOROLE, 2012; NICOLICH; MONTENEGRO, 1997; BERNARDINO JUNIOR et al., 2008; STEVÃO, 2015; MATARASSO, 2006). Normalmente, rostos arredondados, constituem as características morfológicas do padrão braquicefalico associada a uma musculatura forte e mais desenvolvida do masseter (CABRERA, 2004; GUEDES; TEIXEIRA; CATTONI, 2010). Nesse sentido, um estudo realizado por Tarallo et al. (2018) objetivou identificar a indicação correta para sua remoção e corresponder melhor às expectativas dos pacientes. Entre 2012 e 2016, pacientes que procuravam melhorar o contorno malar por redução da proeminência submalar foram incluídos no estudo, foi solicitada uma ressonância magnética pré-operatória para identificar corretamente o volume da gordura de Bichat ou a presença de hipertrofia do musculo masseter; foram oferecidas aos pacientes dúas opções de tratamento: remocao da gordura de Bichat e injeção de 50 UI de toxina botulínica. Concluiram que não existe uma abordagem adequada para todos os casos, pois os dois grupos ficaram satisfeitos com os resultados. Os critérios visuais sugeridos, o exame clínico e a análise de imagem são úteis no estabelecimento da condição do paciente e na determinação dos métodos de tratamento apropriados para melhorar o perfil facial (TARALLO et al., 2018).

Feng et al. (2019), tentando identificar a influência do volume do terço médio na estética facial avaliada por ortodontistas e não especialistas, realizaram uma pesquisa onde mostraram aos avaliadores uma imagem natural e outra do sorriso de uma mulher de 25 anos com face equilibrada que foi modificada em diversos graus de decréscimo e acréscimo na bochecha. Concluiram que, existem diferenças entre ortodontistas e não especialistas na percepção da estética da bochecha e que a influência do volume do terço médio para diagnostico em ortodontia necessita de maiores considerações pois não chegaram a um censo comum.

É imperioso ressaltar ainda, que com o tempo a bola de Bichat gradualmente perde volume, o que levaria a uma face envelhecida com a necessidade de usar preenchimentos ou implantes a fim de dar uma aparência de plenitude facial e jovialidade (LOUKAS et al., 2006; TARALLO et al., 2018; BRAZ; SAKUMA, 2012).

Estudos recentes, ponderam que apesar da multiplicidade de usos clínicos e estéticos, as indicações corretas para a remoção da gordura de Bichat ainda não foram totalmente elucidadas. De fato, a gordura de Bichat involui com a idade e sua remoção pode levar a um afinamento excessivo da face que pode falhar em proporcionar uma aparência jovem, especialmente considerando que o uso de enxerto de gordura é atualmente um dos procedimentos mais comuns para rejuvenescimento facial (TARALLO et al., 2018).

A literatura não é unanime em relação a indicação da bichectomia em todas as idades. Alguns profissionais realizam o procedimento a fim de esculpir os ângulos faciais para alcançar o triângulo invertido da juventude. Na outra extremidade temse o uso de preenchimentos e implantes para pacientes que desejam adicionar contorno e projeção, ou seja, volume, em áreas atróficas devido ao envelhecimento normal da face. A dúvida iminente é: para que retirar a gordura de Bichat se com o envelhecimento precisa-se de volume? Matarasso (1991) relata que existe uma fraca relação entre gordura corporal e tamanho da bola de Bichat, e mesmo com o envelhecimento e a perda característica de gordura, a bola de Bichat permanece em um tamanho relativamente fixo, demostrando resistência a lipólise. No entanto, é importante destacar que há falta de conhecimento em relação aos efeitos em longo prazo do procedimento e seu papel no envelhecimento facial (TAPIA; RUIZ-DE-ERENCHUN; RENGIFO, 2006; KRUPP, 1986).

Uma avaliação global do tecido mole do paciente de frente e perfil é essencial para que se tenha um entendimento amplo 
das características estéticas e de quais indicações seriam necessárias para ele a fim de obter sucesso em procedimentos estéticos tanto bichectomia como preenchimentos.

\section{Conclusão}

A bichectomia deve ser preconizada para melhorar a função mastigatória em detrimento apenas do desígnio estético, uma vez que as consequências desse procedimento são incertas com o evolver da idade.

\section{Referências}

AHARI, U.Z. et al. The buccal fat pad: importance and function. IOSR J. Dent. Med. Scie., v.12, n.6, p.79-81, 2016. doi: 10.9790/0853-15060137981

BERGMAN, R.T. Cephalometric soft tissue facial analysis. Am. J. Orthod. Dentofacial Orthop., v.116, n.4, p.373-89, 1999. doi: 10.1016/s0889-5406(99)70222-2

BERNARDINO JUNIOR, R. et al. Corpo adiposo da bochecha: um caso de variação anatômica. Biosc. J., v.24, n.4, p.108-13, 2008.

BRAZ,A.V.; SAKUMA, T.H. Midface rejuvenation: an innovative technique to restore cheek volume. Dermatol. Surg., v.38, n.1, p.118-120, 2012. doi: 10.1111/j.1524-4725.2011.02161.x

CABRERA, C.A.G. Ortodontia clínica. Curitiba: Produções Interativas, 2004

CADENA, S.M.D.; GUERRA, C.M.F. Aparência facial e a imagem ideal. Rev. Dent. Press Estét, v.3, n.1, p.27-38, 2006.

CAMARA, C.A. Esthetics in Orthodontics: interest points, reference points and discrepancy points. Dent. Press J. Orthod., v.17, n.5, p.4-7, 2012. doi: 10.1590/S2176-94512012000500003

CERRATI, E.W.; THOMAS, J.R. The multicultural evolution of beauty in facial surgery. Braz. J. Otorhinolaryngol., v.83, n. 4, p. 373-374, 2017. doi: 10.1016/j.bjorl.2017.04.005

COHEN, S.R. et al. Buccal fat pad augmentation for facial rejuvenation. Plast. Reconstr. Surg., v. 139, p.1273-1276, 2017. doi: 10.1097/PRS.0000000000003384

CONTI, G. et al. Regenerative potential of the Bichat fat pad determined by the quantification of multilineage differentiating stress enduring cells. Eur. J. Histochem., v.62, n.4, p.2900, 2018. doi: 10.4081/ejh.2018.2900

DEAN, A. et al. The buccal fat pad flap in oral reconstruction. Head Neck, v.23, p.383-388, 2001. doi: 10.1002/hed.1048

DUBIN, B. et al. M. Anatomy of the buccal fat pad and its clinical significance. Plast Reconstr. Surg., v. 83, n. 2, p. 257-64, 1989. doi: 10.1097/00006534-198902000-00009

EL-KEHDY, J.; ABBAS, O.: RUBEIZ, N. A review of ParryRomberg syndrome. J. Am. Acad. Dermatol., v. 67, n. 4, p. 769784, 2012. doi: 10.1016/j.jaad.2012.01.019

ENGDAHL, R. et al. Superselective microcatheter embolization of hemorrhage after buccal lipectomy. Aesthetic Plast Surg., v. 36, n. 3, p. 742-5, 2012. doi: 10.1007/s00266-012-9878-1

FARIA, C.A.C. et al. Bichetomia e sua contribuição para harmonia facial. Rev. Bras Cir Plást., v.33, n.4, p.446-452, 2018.

FENG, J. et al. Esthetic evaluation of facial cheek volume: A study using 3D stereophotogrammetry. Angle Orthod., v.89, n.1, p.129-137, 2019. doi: 10.2319/020418-97.1

GAUGHRAN, G.R.L. Fasciae of the masticator space. Anat.
Rec., v.129, p.383-399, 1957. doi: 10.1002/ar.1091290403

GUEDES, S.P.C.; TEIXEIRA, B.V.; CATTONI, D.M. Orofacial measurements in teenagers from Rio de Janeiro State according to facial typology. Rev. CEFAC, v.12, n.1, p 68-74, 2010. doi: 10.1590/S1516-18462009005000058

KOŚCIŃSKI, K. Facial attractiveness: General patterns of facial preferences. Anthropol. Rev., v.70, p. 45-79, 2007. doi: 10.2478/ v10044-008-0001-9

KRUPP, S. Buccal lipectomy - reappraisal and case report. Eur. J. Plast. Surg., v. 9, n. 1, p. 40-2, 1986. doi: 10.1007/BF00294797

LI, W.T.; XU, D.C.; ZHONG, S.Z. Applied anatomy of the buccal fat pad transposition for the treatment of soft tissue defects in the maxillofacial region. Chin. J. Clin. Anat, v.11, n.1, p.165-170, 1993

LOUKAS, M. et al. Gross anatomical, CT and MRI analyses of the buccal fat pad with special emphasis on volumetric variations. Surg Radiol Anat., v.28, n.254-60, 2006. doi: 10.1007/s00276006-0092-1

MATARASSO, A. Buccal fat fad excision: aesthetic improvement of the midface. Ann Plast Surg., v. 26, n. 5, p. 413-38, 1991. doi: 10.1097/00000637-199105000-00001

MATARASSO, A. Pseudoherniation of the buccal fat pad: a new clinical syndrome. Plast. Reconstr. Surg., v.112, n.6, p.1716-20, 2003. doi: 10.1097/01.PRS.0000086080.81468.4A

MATARASSO, A. Managing the buccal fat pad. Aesthet Surg J., v.26, n.3, p.330-336, 2006. doi: 10.1016/j.asj.2006.03.009

MOROSINI, I. A. C et al. Estudo da agradabilidade da face utilizando a análise facial frontal em fotografias padronizadas. Rev. Dental Press J Orthod., v. 17, n.5, p.24-34, 2012

MUESER, K. et al. You are only as pretty as you feel: Facial expression as a determinant of physical attractiveness. $J$. Personal. Soc. Psychol., v.46, n.2, p.469-478, 1984.

NAINI, F.B.; MOSS, J.P.; GILL, D.S. The enigma of facial beauty: Esthetics, proportions, deformity, and controversy. Am. J. Orthod. Dentofacial Orthop., v.130, n.3, p.277-82, 2006. doi: 10.1016/j.ajodo.2005.09.027

NICOLICH, F.; MONTENEGRO, C. Extracción de la bola de Bichat: una operación simple con sorprendentes resultados. Folia Dermatol Peru, v. 8, n. 1, p. 1-5, 1997.

NORMANDO, D. A estética e a percepção humana. Dental Press J. Orthod., v.17, n.5, p.1, 2012. doi: 10.1590/S217694512012000500001

RACZ, L.; MAROS, T.N.; SERES-STURM, L. Structural characteristics and functional significance of the buccal fat pad (corpus adiposum buccae). Morphol. Embryol. (Bucur), v 35, n.2, p.73-77, 1989.

REIS, S.A.B. et al. Avaliação dos fatores determinantes da estética do perfil facial. Rev. Dental Press Ortodon. Ortop. Facial, v.16, n.11, p.57-67, 2011. doi: 10.1590/S2176-94512011000100010

RHODES, G. The evolutionary psychology of facial beauty. Annu Rev. Psychol., v.57, p.199-226, 2006. doi: 10.1146/annurev. psych.57.102904.190208

SILVA et al. Bichectomy as facial aesthetic procedure: prospective observational study. J. Braz. Coll. Oral. Maxillofac. Surg., v.5, n.3, p.17-23, 2019. doi: 10.14436/2358-2782.5.3.017-02.3.oar

SILVA, R.M.A.F; SILVA FILHO, J.P. Avaliação dos contornos faciais após remoção da bola de Bichat: revisão de literatura. RFAIPE, v.7, n.2, p.73-84, 2017.

STEVÃO, E.L.L. Bichectomy or bichatectomy: small and simple 
intraoral surgical procedure with great facial results. $A d v$. Dent Oral Health, v.1, n.1, p.1-4, 2015

STUZIN, J.M. et al. The anatomy and clinical applications of the buccal fat pad. Plastic Reconstructive Surg., v.85, n.1, p.29-37, 1990. doi: 10.1097/00006534-199001000-00006

TAPIA, A.; RUIZ-DE-ERENCHUN. R.; RENGIFO, M. Combined approach for facial contour restoration: treatment of malar and cheek areas during rhytidectomy. Plast Reconstr Surg., v.118, n.2, p.491-497, 2006. doi: 10.1097/01. prs.0000235265.26138.66

TARALLO, M. et al. Clinical significance of the buccal fat pad: how to determine the correct surgical indications based on preoperative analysis. Int. Surg. J., v.5, n.4, p.1192-1194, 2018. doi: 10.18203/2349-2902.isj20181100

TATARUNAITE, E. et al. Facial attractiveness: a longitudinal study. Am. J. Orthod. Dentofacial Orthop., v.127, n.6, p 676-82, 2005. doi: 10.1016/j.ajodo.2004.01.029

TEDESCO, L.A. et al. A dental-facial attractiveness scale. Am. J. Orthod. Dentofacial Orthop., v.83, n.1, p.38-43, 1983. doi: 10.1016/0002-9416(83)90269-5

THOMAS, M.K.; D'SILVA, J.A.; BOROLE, A.J. Facial sculpting: Comprehensive approach for aesthetic correction of round face. Indian J. Plast. Surg., v.45, n.1, p.122-7, 2012. doi: 10.4103/0970-0358.96608

TIDEMAN, H.; BOSANQUET, A.; SCOTT, J. Use of the buccal fat pad as a pedicled graft. J. Oral. Maxilofac. Surg., v.44, p.435440, 1986. doi: 10.1016/s0278-2391(86)80007-6

TOSTEVIN, P.M.; ELLIS, H. The buccal pad of fat: a review. Clin. Anat., v.8, n.6, p.403-406, 1995. doi: 10.1002/ca.980080606

WILSON, W. L. A critical analysis of orthodontic concepts and objectives. Am. J. Orthod., St. Louis, p. 891-918, 1957. doi: 10.1016/0002-9416(57)90150-1

YOUSIF, N.J. et al. The nasolabial fold: an anatomic and histologic reappraisal. Plast Reconstr Surg., v.93, n.1, p.60-69, 1994.

YOUSUF, S. et al. A review of the gross anatomy, functions, pathology, and clinical uses of the buccal fat pad. Surg Radiol Anat, v. 32 , n. 5, p. 427-436, 2010. doi: 10.1007/s00276-0090596-6

ZHANG, H.M. et al. Anatomical structure of the buccal fat pad and its clinical adaptations. Plast Reconstr Surg., v. 109, n. 7, p. 2509-2518, 2002. doi: 10.1097/00006534-200206000-00052 